\title{
ПЕРЕВОДНЫЕ СТАТЬИ
}

УДК: 531.384, 517.925

MSC 2010: 70F25, 37J60, 70E18

\section{Интеграл Якоби в неголономной механике*}

\author{
А. В. Борисов, И. С. Мамаев, И. А. Бизяев
}

В работе обсуждаются условия существования интеграла Якоби (обобщающего энергию) в системах с неоднородными и неголономными связями. В качестве примера подробно рассмотрена задача о движении саней Чаплыгина на вращающейся плоскости и движение динамически симметричного шара на равномерно вращающейся поверхности. Кроме того, обсуждаются наглядные механические демонстрации, основанные на движении однородного шара на вращающемся столе и на поверхности Бельтрами.

Ключевые слова: неголономная связь, интеграл Якоби, сани Чаплыгина, вращающийся стол, задача Суслова \footnotetext{
and Chaotic Dynamics, 2015, vol. 20, no. 3, pp. 383-400.

Получено 28 апреля 2015 года

После доработки 13 мая 2015 года вузам. Работа И. А. Бизяева поддержана грантом РФФИ № 15-38-20879 мол_а_вед.

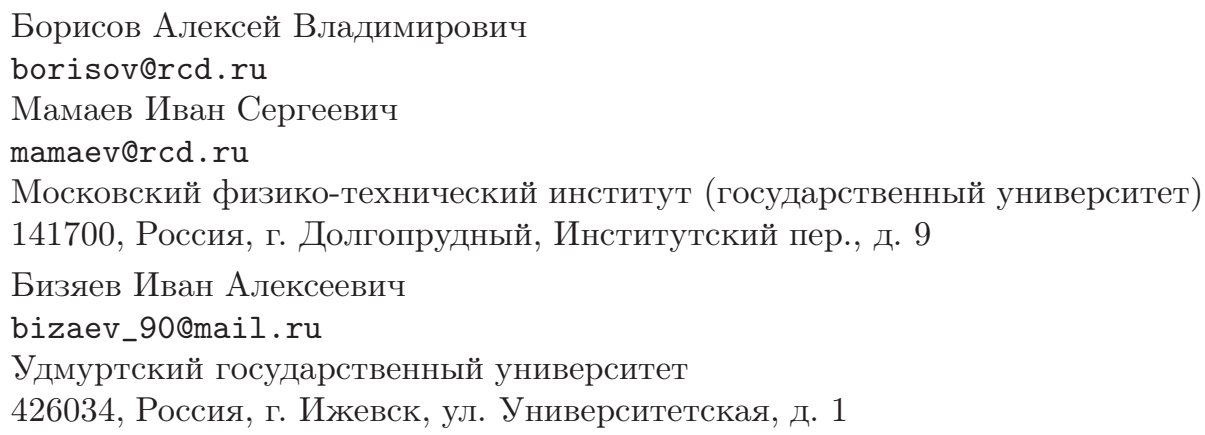

*Перевод статьи "The Jacobi Integral in Nonholonomic Mechanics", опубликованной в журнале Regular

Работа А. В. Борисова, И. С. Мамаева выполнена в рамках базовой части государственного задания 


\section{Введение}

Неинтегрируемые связи в неголономной механике, имеющие естественные механические реализации, представляются в форме

$$
\sum_{i=1}^{n} a_{\mu i}(\boldsymbol{q}) \dot{q}_{i}-b_{\mu}(\boldsymbol{q})=0, \quad \mu=1, \ldots, m, \quad m<n,
$$

где $\boldsymbol{q}=\left(q_{1}, \ldots, q_{n}\right), q_{i}$ - обобщенные координаты. Еще чаще встречается случай $b_{i}(\boldsymbol{q}) \equiv 0$, то есть случай линейных однородных связей (при $b_{i}(\boldsymbol{q}) \not \equiv 0$ связи неоднородны). Механические системы с однородными связями хорошо известны: качение тел друг по другу без проскальзывания, скольжения колесиков, лезвий (и пр.) без поперечного смещения или различные их комбинации. Динамика таких систем изучена, например, в [4, 13, 18, 20, 33]. Случай $b_{i}(\boldsymbol{q})=$ const $\neq 0$ формально рассматривался в задаче Суслова [3] и Веселовой [11] без построения механической модели, но для апробации различных методов интегрирования и качественного анализа.

Наиболее естественный пример линейных неоднородных связей возникает при описании качения без проскальзывания твердых тел по поверхностям, вращающимся с постоянной угловой скоростью вокруг некоторой оси, неподвижной в пространстве. Например, качение однородного шара по равномерной вращающейся плоскости (по вращающемуся столу - turntable) было, видимо, впервые исследовано С. Ирншоу (см. его учебник по динамике 1844 года [21]) еще до появления неголономной механики. Он показал, что траектории центра шара в абсолютном пространстве представляют собой окружности, положение центра которых зависит от начальных условий. Та же задача обсуждается позже в учебниках Е. Милна (1948 г.) [22] и Ю. И. Неймарка и Н. А. Фуфаева (1967 г.) [5]. Э. Раус в своем трактате [9] в одном из примеров сводит задачу о качении к задаче о скольжении под действием некоторой гироскопической силы, касательной к траектории. Множество публикаций по этой задаче Ирншоу, выполненных на элементарном уровне, имеется в American Journal of Physics (AJP). В этих работах обсуждаются также гироскопическая (электромагнитная) аналогия, восходящая к Раусу [23], влияние трения, наклона и кривизны стола [24, 27, 29], неоднородности угловой скорости вращения, зависящей от расстояния до центра стола [25]. В работе [25] обсуждается известная демонстрация в музее Франклина, в которой катящийся по прямой шар попадает на вращающийся стол, а потом покидает его по той же прямой (то есть генерируемое здесь отображение рассеяния является тождественным, см. [32]). В работе [26] поставлена (но не решена) задача о движении однородной вертикальной монеты по вращающемуся столу, вводится модель трения качения и предлагается биллиардная задача для случая, когда стол ограничен круговой стенкой. Отметим также работы Н. А. Фуфаева [30, 31], посвященные анализу движения шара по вращающейся плоскости и сфере. Для случая плоскости введены различные модели трения и получены весьма противоречивые результаты, которые требуют экспериментального изучения (см. также [24]). Для случая качения по вращающейся сфере в поле тяжести в [30] указана аналогия с движением волчка Лагранжа. Эта аналогия обсуждается также у Рауса [9] и Фиркандта [37], а явное преобразование, исключающее вращение сферы, указано в [4].

В общем случае задача о качении однородного шара по равномерно вращающейся осесимметричной поверхности впервые обсуждается в работе [4], в основном посвященной случаю неподвижной поверхности (см. также [34]). Однако, как отмечено в недавней работе [1], для интегрируемости уравнений движения шара на поверхности вращения в [4] не хватает некоторого аналога интеграла энергии (названного в [1] moving energy). Отметим, что 
для неоднородных связей существование интеграла энергии, вообще говоря, не очевидно и в общем случае он отсутствует. В [1] сформулировано утверждение о его существовании в случае небольших скоростей вращения поверхности.

В этой работе мы пытаемся прояснить механический смысл возникновения такого интеграла (названного нами интегралом Якоби) для случая произвольной, не обязательно осесимметричной поверхности, указываем его явно для качения шара по поверхности вращения, а также для новой задачи о движении саней Чаплыгина по вращающейся плоскости (частный случай этой задачи, эквивалентной качению вертикальной однородной монеты по вращающейся плоскости, был рассмотрен в [38] с применением методов геометрической механики, поэтому результаты сложно понять и верифицировать). Мы получаем также общие условия существования интеграла Якоби для неоднородных связей, а в (неоднородной) задаче Суслова указываем условия на параметры системы, при которых он существует (но имеет теперь скрытый характер).

В заключение отметим опубликованную после сдачи нашей статьи в печать работу [2], в которой обсуждаются условия существования интеграла энергии.

\section{1. Общий формализм}

\section{1. Интеграл энергии в неголономных системах}

Как хорошо известно в неголономной механике (см., например, [7]), если силы, действующие на систему, обобщенно потенциальны (то есть представляются в виде суммы потенциальных и гироскопических сил), функция Лагранжа не зависит явно от времени, а линейные неинтегрируемые связи однородны по скоростям, то неголономная система допускает интеграл энергии.

Действительно, запишем уравнения движения с неопределенными множителями для системы со связями

$$
\begin{gathered}
\left(\frac{\partial L}{\partial \dot{q}_{i}}\right)^{\cdot}-\frac{\partial L}{\partial q_{i}}=\sum_{\mu} \lambda_{\mu} \frac{\partial f_{\mu}}{\partial \dot{q}_{i}}, \quad i=1, \ldots, n, \mu=1, \ldots, m, \\
f_{\mu}(\boldsymbol{q}, \dot{\boldsymbol{q}})=0,
\end{gathered}
$$

где $q_{i}, \dot{q}_{i}$ - обобщенные координаты и скорости, $\lambda_{\mu}$ - неопределенные множители, $f_{\mu}-$ однородные по $\dot{q}_{i}$ функции, $L(\boldsymbol{q}, \dot{\boldsymbol{q}})-$ функция Лагранжа.

Интеграл энергии имеет стандартную форму

$$
E=\sum_{i} \dot{q}_{i} \frac{\partial L}{\partial \dot{q}_{i}}-L
$$

Дифференцируя $E$ в силу системы (1.1) и применяя формулу Эйлера для однородных функций, получим искомый закон сохранения:

$$
\dot{E}=\sum_{\mu, i} \lambda_{\mu} \frac{\partial f_{\mu}}{\partial \dot{q}_{i}} \dot{q}_{i}=\kappa \sum_{\mu} \lambda_{\mu} f_{\mu}=0,
$$

где $\kappa-$ степень однородности.

Пусть теперь линейные связи неоднородны по скоростям:

$$
f_{\mu}=\sum_{i} a_{\mu i}(\boldsymbol{q}) \dot{q}_{i}-b_{\mu}(\boldsymbol{q})=0, \quad \mu=1, \ldots, m .
$$


Такие связи еще называются аффинными. Тогда производная энергии (1.2) вдоль системы (1.1) после ограничения на связь представится в форме

$$
\dot{E}=\sum \lambda_{\mu} b_{\mu}(\boldsymbol{q})
$$

то есть в общем случае энергия (1.2) не является первым интегралом.

ЗАмечАНИЕ. В только что появившихся работах $[1,2]$ исследуются условия, при которых система с неоднородными связями (1.4) допускает интеграл энергии (1.2).

\section{2. Вращение опорной поверхности и интеграл Якоби}

Имеется важный частный случай неоднородных связей, когда система допускает обобщение интеграла энергии (1.2): это случай качения твердого тела и различных механизмов, подчиненных неголономным связям, по поверхности, которая равномерно вращается. Как хорошо известно, переход в равномерно вращающуюся (неинерциальную) систему отсчета эквивалентен добавлению к лагранжиану системы обобщенно потенциальных (линейных по скоростям) и потенциальных (зависящих только от координат) членов, отвечающих кориолисовым и центробежным силам соответственно. При этом связь в этой системе оказывается однородной, и поэтому существует интеграл вида (1.2), который мы будем называть интегралом Якоби, используя аналогию с небесной механикой (круговой ограниченной задачей трех тел). Таким образом, справедливо

Предложение 1. Пусть движение некоторой механической системы по твердой неподвижной (в некоторой инерциальной системе координат) поверхности описывается лагранжевой системой с однородными линейными по (обобщенным) скоростям связями, тогда система обладает интегралом энергии. Если та же система движется по той же, но равномерно вращающейся, поверхности, то она также допускает интеграл Якоби.

Как было сказано выше, доказательство предложения 1 не требует вычислений и апеллирует к хорошо известным фактам механики.

ЗАмечАниЕ. Связи могут быть как голономными, так и неголономными, и, в частности, интеграл Якоби существует и для абсолютно гладкой, и для абсолютно шероховатой опорных поверхностей, причем не обязательно осесимметричных. Ниже мы приведем явный вид интеграла Якоби для качения однородного шара и саней Чаплыгина по равномерно вращающейся поверхности.

ЗАмечАниЕ. Как уже отмечалось во введении, наблюдение, что равномерное вращение плоскости и сферы и неголономные связи приводят к появлению дополнительных гироскопических слагаемых в лагранжиане задачи с идеальным скольжением, восходит еще к Раусу [9].

\section{3. Обобщение интеграла Якоби}

В принципе (локально) любую связь вида (1.4) при помощи замены координат, явно зависящей от времени, можно сделать однородной. Для этого необходимо выбрать замену координат $\boldsymbol{q}=\boldsymbol{q}(\boldsymbol{Q}, t)$, удовлетворяющую уравнению

$$
\frac{\partial \boldsymbol{q}}{\partial t}=\boldsymbol{v}_{0}(\boldsymbol{q})
$$

где $\boldsymbol{v}_{0}(\boldsymbol{q})$ - некоторое частное решение уравнений связи (1.4), а $\boldsymbol{Q}$ - новые локальные координаты, которые являются первыми интегралами системы (1.5). Однако при этом в общем случае при переходе к новым координатам получим функцию Лагранжа $L(\boldsymbol{Q}, \dot{\boldsymbol{Q}}, t)=$ $=L\left(\boldsymbol{q}(\boldsymbol{Q}, t), \frac{\partial \boldsymbol{q}}{\partial t}+\frac{\partial \boldsymbol{q}}{\partial \boldsymbol{Q}} \dot{\boldsymbol{Q}}\right)$, которая явно содержит время $t$, поэтому энергия не сохраняется. 
Предложение 2. Если для неоднородной связи (1.4) существуют частные решения уравнений (1.4) $\dot{\boldsymbol{q}}=\boldsymbol{v}_{0}(\boldsymbol{q})$ и неавтономная замена $\boldsymbol{q} \rightarrow \boldsymbol{Q}$, такие, что выполнено соотношение

$$
\frac{\partial \boldsymbol{q}(\boldsymbol{Q}, t)}{\partial t}=\boldsymbol{v}_{0}(\boldsymbol{q}(\boldsymbol{Q}, t))
$$

и при этом функиия Лагранжа в новых переменных $\boldsymbol{Q}, \dot{\boldsymbol{Q}}$ не зависит явно от времени, то система (1.1) обладает (обобщенным) интегралом Якоби.

В качестве примера укажем задачу Суслова с неоднородной связью, существование интеграла Якоби для которой, вообще говоря, не типично [3], а неоднородность приводит к тому, что на фазовом портрете инерциального движения появляется фокус, приводящий к отсутствию аналитического интеграла. Движение твердого тела с неподвижной точкой в рассматриваемой задаче задается функцией Лагранжа

$$
L=\frac{1}{2}(\boldsymbol{\omega}, \mathbf{I} \boldsymbol{\omega})-U(\boldsymbol{q}),
$$

где $\boldsymbol{\omega}$ - угловая скорость в осях, жестко связанных с телом, $U(\boldsymbol{q})$ - потенциальная энергия, неоднородная связь Суслова определяется уравнением

$$
\omega_{3}=c, \quad c=\text { const. }
$$

Сразу отметим, что корректная механическая реализация такой связи при $c \neq 0$ пока неизвестна (при $c=0$ она указана Вагнером, но не обобщается на $c \neq 0$; см. подробнее [10]).

Воспользуемся в качестве обобщенных координат углами Эйлера. Тогда

$$
\begin{gathered}
\omega_{1}=\dot{\psi} \sin \theta \sin \varphi+\dot{\theta} \cos \varphi, \quad \omega_{2}=\dot{\psi} \sin \theta \cos \varphi-\dot{\theta} \sin \varphi \\
\omega_{3}=\dot{\psi} \cos \theta+\dot{\varphi} .
\end{gathered}
$$

Отсюда мы видим, что одна из возможных замен переменных, приводящая связь к однородному виду, может быть записана как

$$
\varphi=c t+\widetilde{\varphi}
$$

Если в этом случае тензор инерции оказывается диагональным и два его главных момента инерции равны $I_{1}=I_{2}$,

$$
\mathbf{I}=\operatorname{diag}\left(I_{1}, I_{1}, I_{3}\right),
$$

а потенциал зависит только от $\theta, \psi$, то функция Лагранжа после замены оказывается автономной:

$$
L=\frac{1}{2} I_{1}\left(\dot{\theta}^{2}+\dot{\psi}^{2}\right)+\frac{1}{2} I_{3}(c+\dot{\tilde{\varphi}})^{2}-\frac{1}{2}\left(I_{1}-I_{3}\right) \cos ^{2} \theta \dot{\psi}^{2}+I_{3} \dot{\psi}(c+\dot{\tilde{\varphi}})-U(\theta, \psi) .
$$

Следовательно, система обладает обобщенным интегралом Якоби вида

$$
\begin{aligned}
E & =\frac{1}{2} I_{1}\left(\dot{\theta}^{2}+\dot{\psi}^{2}\right)+\frac{1}{2} I_{3}(c+\dot{\widetilde{\varphi}})^{2}-\frac{1}{2}\left(I_{1}-I_{3}\right) \cos ^{2} \theta \dot{\psi}^{2}+ \\
& +I_{3} \dot{\psi}(c+\dot{\tilde{\varphi}})+c(c+\dot{\widetilde{\varphi}}+\cos \theta \dot{\psi})+U(\theta, \psi) .
\end{aligned}
$$

Например, в случае поля тяжести $U=m g \cos \theta$ задача является интегрируемой вследствие существования циклических интегралов и представляет собой одно из возможных неголономных обобщений задачи о волчке Лагранжа. Наличие интеграла Якоби в этой ситуации

НЕЛИНЕЙНАЯ ДИНАМИКА. 2015. Т. 11. № 2. С. 377-396 
имеет скрытый характер и требует дополнительной симметрии у системы. Для системы Веселовой (родственной задаче Суслова и отличающейся от нее тем, что теперь фиксируется проекция угловой скорости на ось, неподвижную в пространстве) интеграл Якоби будет существовать при любых параметрах системы. Несмотря на свой скрытый характер, еще в [36] он был явно обозначен как интеграл Якоби-Пенлеве, хотя и не связан непосредственно с равномерным вращением системы координат. Его существование можно обосновать с помощью предложения 2. Гамильтонизация системы Веселовой с неоднородной связью обсуждается в работе [11].

\section{2. Сани Чаплыгина на вращающейся плоскости}

\section{1. Уравнения движения. Первые интегралы}

Напомним, что сани Чаплыгина (см., например, [13]) представляют собой твердое тело, опирающееся на плоскость двумя абсолютно гладкими ножками и острым невесомым колесиком (диском или лезвием), которое препятствует проскальзыванию его точки контакта $P$ в перпендикулярном плоскости колесика направлении (см. рис 1). Обозначим точкой $C$ положение центра масс всей системы и рассмотрим движение саней Чаплыгина по плоскости, которая равномерно вращается. Движение саней подробно изучено не только для неподвижной горизонтальной [12], но и для неподвижной наклонной плоскости [13].

Определим три системы координат (см. рис. 1):

- инерциальную (неподвижную) систему координат $O x_{o} y_{o}$,

- неинерциальную систему координат $O x y$, которая вращается относительно инерциальной с постоянной угловой скоростью $\Omega$,

- неинерциальную систему координат $O_{1} x_{1} x_{2}$, жестко связанную с санями Чаплыгина и началом отсчета $O_{1}$, которое расположено на пересечении перпендикуляра к плоскости лезвия в точке контакта $P$ и прямой, параллельной плоскости лезвия и проходящей через центр масс $C$.

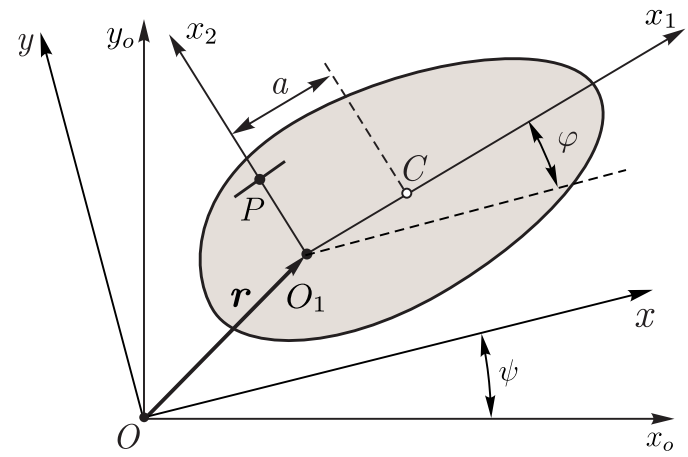

Рис. 1. Сани Чаплыгина на вращающейся плоскости.

Конфигурационное пространство $\mathcal{N}$ в данном случае совпадает с группой движений плоскости $S E(2)$; для его параметризации выберем декартовы координаты $(x, y)$ точки $O_{1}$ в системе координат $O x y$ и угол $\varphi$ поворота осей $O_{1} x_{1} y_{1}$ относительно $O x y$. 
Пусть $\boldsymbol{v}_{o}$ и $\omega_{0}-$ (абсолютная) скорость точки $O_{1}$ и угловая скорость тела в системе координат $O x_{o} y_{o}$ соответственно. Тогда связь, выражающая условие равенства нулю проекции скорости точки $P$ на ось $O_{1} x_{2}$, имеет вид

$$
\left(\boldsymbol{v}_{o}+\Omega \mathbf{J}_{n} \boldsymbol{r}, \boldsymbol{e}_{2}\right)=0, \quad \mathbf{J}_{n}=\left(\begin{array}{cc}
0 & -1 \\
1 & 0
\end{array}\right)
$$

где $\boldsymbol{r}$ - вектор, соединяющий точки $O$ и $O_{1}$, а $\boldsymbol{e}_{1}, \boldsymbol{e}_{2}$ здесь и далее в разделе обозначают единичные векторы осей $O_{1} x_{1}, O_{1} x_{2}$ соответственно.

Кинетическая энергия представляется в форме

$$
T=\frac{1}{2} m \boldsymbol{v}_{o}^{2}+\frac{1}{2} I \omega_{o}^{2}+m a \omega_{o}\left(\boldsymbol{v}_{o}, \boldsymbol{e}_{2}\right)
$$

где $m$ - масса тела, а $I$ - его момент инерции относительно точки $O_{1}$.

ЗАмЕчАниЕ. Произведение $\Omega \mathbf{J}_{n} \boldsymbol{r}$ эквивалентно проекции на плоскость векторного произведения $\boldsymbol{\Omega} \times \boldsymbol{r}$.

Для перехода в неинерциальную систему координат $O x y$ выполним преобразование

$$
\boldsymbol{v}_{o}=\boldsymbol{v}+\Omega \mathbf{J}_{n} \boldsymbol{r}, \quad \omega_{o}=\omega+\Omega,
$$

где $\boldsymbol{v}=\left(v_{1}, v_{2}\right)$ - скорость точки $O_{1}$ в системе координат $O_{1} x_{1} x_{2}$, а $\omega-$ угловая скорость тела относительно плоскости:

$$
v_{1}=\dot{x} \cos \varphi+\dot{y} \sin \varphi, \quad v_{2}=-\dot{x} \sin \varphi+\dot{y} \cos \varphi, \quad \omega=\dot{\varphi} .
$$

В результате связь (2.1) перепишется в форме

$$
v_{2}=0
$$

Функцию Лагранжа можно представить в виде

$$
\begin{aligned}
L & =\frac{1}{2} m\left(v_{1}^{2}+v_{2}^{2}\right)+\frac{1}{2} I \omega^{2}+m a(\omega+\Omega) v_{2}+m a \Omega \omega x_{1}+ \\
& +m \Omega\left(x_{1} v_{2}-x_{2} v_{1}\right)+\frac{1}{2} m \Omega^{2}\left(\left(x_{1}+a\right)^{2}+x_{2}^{2}\right)-U,
\end{aligned}
$$

где $U-$ потенциальная энергия внешних сил. Кроме того, в $L$ следует полагать $x_{1}$ и $x_{2}$ зависимыми с $(x, y, \varphi)$ :

$$
x_{1}=x \cos \varphi+y \sin \varphi, \quad x_{2}=-x \sin \varphi+y \cos \varphi .
$$

Их эволюция описывается кинематическими соотношениями

$$
\dot{x}_{1}=v_{1}+\omega x_{2}, \quad \dot{x}_{2}=-\omega x_{1} .
$$

Разрешив (2.2) относительно обобщенных скоростей $\dot{\boldsymbol{q}}=(\dot{x}, \dot{y}, \dot{\varphi})$, получим

$$
\dot{\boldsymbol{q}}=v_{1} \boldsymbol{\tau}_{1}+\omega \boldsymbol{\tau}_{2}+v_{2} \boldsymbol{n}
$$


где $\boldsymbol{n}$ - трансверсальное, а $\boldsymbol{\tau}_{1}, \boldsymbol{\tau}_{2}$ - касательные к распределению связей векторные поля:

$$
\boldsymbol{n}=-\sin \varphi \frac{\partial}{\partial x}+\cos \varphi \frac{\partial}{\partial y}, \quad \boldsymbol{\tau}_{1}=\cos \varphi \frac{\partial}{\partial x}+\sin \varphi \frac{\partial}{\partial y}, \quad \boldsymbol{\tau}_{2}=\frac{\partial}{\partial \varphi}
$$

Используя их коммутационные соотношения

$$
\left[\boldsymbol{\tau}_{1}, \boldsymbol{n}\right]=0, \quad\left[\boldsymbol{\tau}_{2}, \boldsymbol{n}\right]=0, \quad\left[\boldsymbol{\tau}_{1}, \boldsymbol{\tau}_{2}\right]=-\boldsymbol{n}
$$

получаем уравнения движения этой системы в общей форме:

$$
\begin{gathered}
\left(\frac{\partial L^{*}}{\partial v_{1}}\right)^{\cdot}-\cos \varphi \frac{\partial L^{*}}{\partial x}-\sin \varphi \frac{\partial L^{*}}{\partial y}=\left(\frac{\partial L}{\partial v_{2}}\right)^{*} \omega \\
\left(\frac{\partial L^{*}}{\partial \omega}\right)^{\cdot}-\frac{\partial L^{*}}{\partial \varphi}=-\left(\frac{\partial L}{\partial v_{2}}\right)^{*} v_{1}
\end{gathered}
$$

В формуле (2.5) фигурирует $L^{*}$ - функция Лагранжа, ограниченная на связь (2.3); отсюда находим

$$
\begin{gathered}
\frac{\partial L^{*}}{\partial v_{1}}=m\left(v_{1}-\Omega x_{2}\right), \quad \frac{\partial L^{*}}{\partial \omega}=I(\omega+\Omega)+m a \Omega x_{1} \\
\left(\frac{\partial L}{\partial v_{2}}\right)^{*}=m a(\omega+\Omega)+m \Omega x_{1} .
\end{gathered}
$$

Учитывая (2.4) и $(2.5)$, окончательно получим уравнения на $\mathcal{M}^{5}=\left\{v_{1}, \omega, x_{1}, x_{2}, \varphi\right\}$ :

$$
\begin{gathered}
m \dot{v}_{1}=m a(\omega+\Omega)^{2}+m \Omega^{2} x_{1}-\frac{\partial U}{\partial x_{1}}, \\
I \dot{\omega}=m a\left(\Omega^{2} x_{2}-(\omega+2 \Omega) v_{1}\right)-\frac{\partial U}{\partial \varphi}, \\
\dot{x}_{1}=v_{1}+\omega x_{2}, \quad \dot{x}_{2}=-\omega x_{1}, \quad \dot{\varphi}=\omega
\end{gathered}
$$

где полагаем, что $U=U\left(x_{1}, x_{2}, \varphi\right)$. Пользуясь соотношением $(1.2)$, получаем для данного случая

$$
E=v_{1} \frac{\partial L^{*}}{\partial v_{1}}+\omega \frac{\partial L^{*}}{\partial \omega}-L^{*}
$$

и находим интеграл Якоби в форме

$$
E=\frac{1}{2}\left(m v_{1}^{2}+I \omega^{2}\right)-\frac{1}{2} m \Omega^{2}\left(\left(x_{1}+a\right)^{2}+x_{2}^{2}\right)+U
$$

Таким образом, справедливо

Предложение 3. Сани Чапльгина на равномерно вращающейся плоскости допускают интеграл Якоби вида (2.7).

В том случае если $U$ не зависит от $\varphi$, в (2.6) отделяется четырехмерная (редуцированная) система, описывающая эволюцию $\left(v_{1}, \omega, x_{1}, x_{2}\right)$. Для ее интегрируемости, по теореме Эйлера - Якоби, не хватает одного дополнительно интеграла и инвариантной меры. Условия существования инвариантной меры обсуждаются в разделе 2.3 . 
Однако для наклонной плоскости потенциал $U$ зависит от $\varphi$. Действительно, если ось $O x$ направлена вдоль линии наибольшего ската, потенциал имеет вид

$$
U=m g \sin \chi\left(x_{1} \sin \varphi+\left(x_{2}+a\right) \cos \varphi\right)
$$

где $\chi$ - угол наклона плоскости к горизонту, a g - ускорение свободного падения. Таким образом, в этом случае нужно исследовать (полную) систему (2.6), которая сохраняет интеграл Якоби.

В случае $U=0$ и $I=m a^{2}$ (то есть когда вся масса системы сосредоточена в точке $C$ ) система (2.10) помимо интеграла Якоби $E$ допускает линейный по скоростям интеграл

$$
F=x_{2} v_{1}-\left(x_{1}+1\right) \omega-\left(\left(x_{1}+1\right)^{2}+x_{2}^{2}\right) .
$$

Однако в этом случае не удается найти инвариантную меру. Отметим, что численные эксперименты показывают отсутствие компактных траекторий в этом случае. Неголономная система с компактными траекториями, обладающая полным набором интегралов, но без инвариантной меры, изучалась в работах $[14,15]$.

\section{2. Суперинтегрируемая динамика уравновешенных саней (конька Чаплыгина)}

Рассмотрим случай, в котором $U=0$ и $a=0$, то есть отсутствует внешнее поле и центр масс всей системы находится на пересечении прямой, проходящей через точку контакта колесика перпендикулярно его плоскости.

В этом случае (2.6) принимает вид

$$
\begin{gathered}
\dot{v}_{1}=\Omega^{2} x_{1}, \quad \dot{\omega}=0, \\
\dot{x}_{1}=v_{1}+\omega x_{2}, \quad \dot{x}_{2}=-\omega x_{1}, \quad \dot{\varphi}=\omega .
\end{gathered}
$$

Система (2.8) обладает стандартной инвариантной мерой, а ее интеграл Якоби представляется в форме

$$
E=\frac{1}{2}\left(m v_{1}^{2}+I \omega^{2}\right)-\frac{1}{2} m \Omega^{2}\left(x_{1}^{2}+x_{2}^{2}\right) .
$$

Кроме того, сохраняется угловая скорость $\omega=$ const и

$$
F=\omega v_{1}+\Omega^{2} x_{2}
$$

Таким образом система (2.8) обладает избыточным набором первых интегралов и, следовательно, является суперинтегрируемой.

Зафиксировав $F=f$ и полагая, что угловая скорость отлична от нуля, получим

$$
v_{1}=\frac{f+\Omega^{2} x_{2}}{\omega}
$$

Отсюда находим траектории на плоскости $x_{1}$ и $x_{2}$ в форме

$$
\left(f+\left(\omega^{2}-\Omega^{2}\right) x_{2}\right)^{2}+\left(\omega^{2}-\Omega^{2}\right) \omega^{2} x_{1}^{2}=h, \quad h=2 E \omega^{2}\left(1-\frac{\omega^{2}}{\Omega^{2}}\right)+f^{2} \frac{\omega^{2}}{\Omega^{2}}
$$

то есть при $\omega^{2}>\Omega^{2}$ получаем семейство эллипсов, а при $\omega^{2}<\Omega^{2}-$ семейство гипербол.

При $\omega=0$ во время движения остаются постоянными $x_{2}$ и $\varphi$, что соответствует прямолинейному равноускоренному скольжению саней вдоль оси $O x_{1}$. 


\section{3. Неподвижные точки и проблема существования инвариантной меры}

Полагая $a$ и $\Omega$ в (2.6) отличными от нуля, изучим препятствия к существованию гладкой инвариантной меры, которые, как известно $[6,16]$, возникают в неподвижных точках фазового потока. Изучим положения равновесия системы (2.6) более подробно. Перейдем к безразмерным переменным (с сохранением предыдущих обозначений)

$$
t \rightarrow \Omega^{-1} t, \quad x_{1} \rightarrow a x_{1}, \quad x_{2} \rightarrow a x_{2}, \quad v_{1} \rightarrow \Omega a v_{1}, \quad \omega \rightarrow \Omega \omega, \quad U \rightarrow m \Omega^{2} a^{2} U,
$$

при этом уравнения движения представятся в виде

$$
\begin{gathered}
\dot{v}_{1}=(\omega+1)^{2}+x_{1}-\frac{\partial U}{\partial x_{1}}, \quad A^{2} \dot{\omega}=x_{2}-(\omega+2) v_{1}-\frac{\partial U}{\partial \varphi}, \\
\dot{x}_{1}=v_{1}+\omega x_{2}, \quad \dot{x}_{2}=-\omega x_{1}, \quad \dot{\varphi}=\omega, \quad A^{2}=\frac{I}{m a^{2}} .
\end{gathered}
$$

Согласно теореме Штейнера,

$$
A^{2}=1+\frac{I_{c}}{m a^{2}}>1
$$

где $I_{c}$ - момент инерции тела относительно центра масс $C$.

Интеграл Якоби с учетом безразмерных переменных (2.9) примет вид

$$
E=\frac{1}{2}\left(v_{1}^{2}+A^{2} \omega^{2}\right)-\frac{1}{2}\left(\left(x_{1}+1\right)^{2}+x_{2}^{2}\right)+U .
$$

Положим $U=0$, тогда система (2.10) имеет два типа неподвижных точек:

1. изолированные положения равновесия

$$
\omega=0, \quad v_{1}=0, \quad x_{1}=-1, \quad x_{2}=0,
$$

2. однопараметрическое семейство положений равновесия

$$
\omega=-1, \quad v_{1}=c, \quad x_{1}=0, \quad x_{2}=c, \quad c=\text { const } .
$$

Положения равновесия второго типа невозможны для гамильтоновых систем. Для неголономных систем такие положения равновесия называются положениями равновесия второго рода, впервые были изучены в работе [17].

Изолированное положение равновесия (2.12) является невырожденной критической точкой интеграла Якоби (2.11). В этом случае центр масс саней совпадает с точкой $O$, при этом сани вращаются вокруг центра масс равномерно с угловой скоростью $\Omega$. Характеристический полином линеаризованной системы в данном случае представляется в форме

$$
P(\lambda)=A^{2} \lambda^{4}+\left(3-A^{2}\right) \lambda^{2}+1 .
$$

Отсюда заключаем, что эта неподвижная точка всегда неустойчива и в зависимости от величины $A^{2} \in[1, \infty)$ может быть одного из двух типов:

- при $1<A^{2}<A_{*}^{2}-$ фокус-фокус,

- при $A_{*}^{2}<A^{2}-$ седло-седло,

где $A_{*}^{2}$ - отличный от единицы корень уравнения $\left(3-A^{2}\right)^{2}-4 A^{2}=0$. В этих положениях равновесия мы не находим препятствий к существованию инвариантной меры. 
Оказывается, семейство (2.13) целиком (то есть при всех значениях $c$ ) лежит на одном из уровней интеграла (2.11), определяемом значением $E=\frac{1}{2}\left(A^{2}-1\right)$. В этом случае сани стоят неподвижно в инерциальной системе координат $O x y$, так что перпендикуляр к лезвию в точке контакта $P$ проходит через точку $O$, а опорная поверхность вращается под санями. Характеристический полином линеаризованной системы имеет вид

$$
P(\lambda)=\lambda^{3}\left(A^{2} \lambda+c\right)
$$

Отсюда, в частности, следует, что $\operatorname{Tr} \mathbf{A}^{(L)}=c$, где $\mathbf{A}^{(L)}$ - матрица линеаризации; следовательно, при $c \neq 0$ не выполняется необходимое условие существования гладкой инвариантной меры, указанное в [6].

Предложение 4. Система (2.10) при $U=0$ не допускает инвариантной меръ с плотностью, аналитической во всем фазовом пространстве.

Поскольку, как мы видели, все данное семейство лежит на одном и том же уровне интеграла Якоби, вопрос о существований или отсутствии инвариантной меры на уровнях интеграла Якоби при $E \neq \frac{1}{2}\left(A^{2}-1\right)$ остается открытым.

Отметим, что компьютерные эксперименты, проведенные А. О. Казаковым, показывают, что при наличии возвращаемости в фазовом потоке (2.10) отсутствуют аттракторы любых типов и система демонстрирует типичное гамильтоново поведение (на двумерном сечении Пуанкаре). Такое поведение для неголономных систем без гладкой инвариантной меры, но обладающих достаточным количеством инволюций уже отмечалось в работе [18].

\section{3. Шар на равномерно вращающейся поверхности}

\section{1. Уравнения движения, интеграл Якоби, инвариантная мера}

Рассмотрим качение без проскальзывания полностью динамически симметричного (в частности, однородного) шара по поверхности, которая вращается вокруг некоторой неподвижной оси с постоянной угловой скоростью $\Omega$.

Определим две системы координат:

- инерциальную (неподвижную) систему координат $O x_{o} y_{o} z_{o}$, в которой ось $O z_{o}$ направлена вдоль оси вращения, то есть $\boldsymbol{\Omega}=(0,0, \Omega)$,

- неинерциальную систему координат $O x_{1} x_{2} x_{3}$, которая вращается с угловой скоростью $\boldsymbol{\Omega}$ относительно $O x_{3}$, причем $O x_{3} \| O z_{o}$.

Вследствие отсутствия проскальзывания в точке контакта $P$, скорость на шаре совпадает со скоростью точки на вращающейся поверхности, то есть

$$
\boldsymbol{f}=\boldsymbol{v}_{o}+a \gamma \times \boldsymbol{\omega}_{o}+\boldsymbol{r}_{p} \times \boldsymbol{\Omega}=0,
$$

где $\boldsymbol{v}_{o}$ и $\boldsymbol{\omega}_{o}-$ скорость центра масс и угловая скорость шара в системе координат $O x_{o} y_{o} z_{o}$, $\boldsymbol{\gamma}$ - нормаль в точке контакта, $a-$ радиус шара, а $\boldsymbol{r}_{p}=O P-$ вектор, направленный из центра масс шара в точку контакта.

Кинетическая энергия шара имеет вид

$$
T=\frac{1}{2} m v_{o}^{2}+\frac{1}{2} I \omega_{o}^{2}
$$

где $m, I$ - масса и момент инерции шара. 


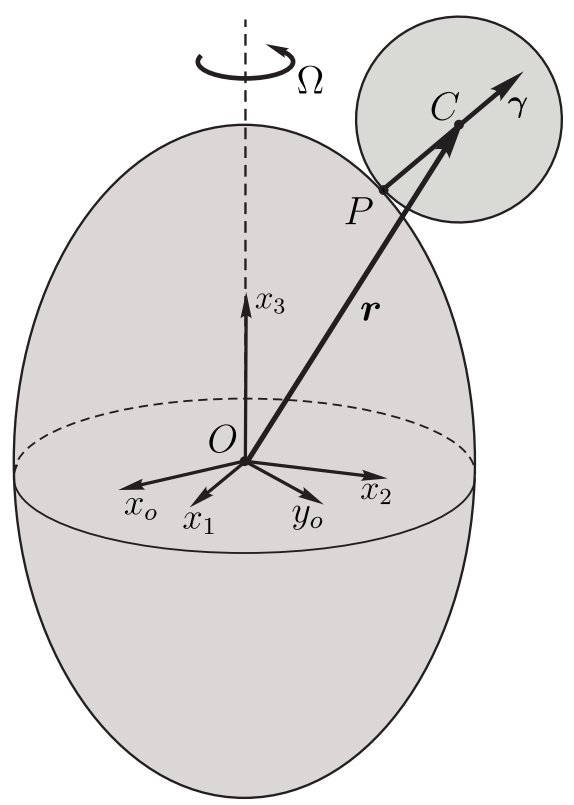

Рис. 2. Шар на вращающейся поверхности.

Далее перейдем в неинерциальную систему координат $O x y z$. Для этого выполним преобразование

$$
\boldsymbol{v}_{o}=\dot{\boldsymbol{r}}+\boldsymbol{\Omega} \times \boldsymbol{r}, \quad \boldsymbol{\omega}_{o}=\boldsymbol{\omega}+\boldsymbol{\Omega},
$$

где $\boldsymbol{r}=\left(r_{1}, r_{2}, r_{3}\right)$ - координаты центра масс шара, а $\boldsymbol{\omega}$ - его угловая скорость в системе координат $O x_{1} x_{2} x_{3}$.

В результате связь станет однородной функцией по скоростям

$$
\boldsymbol{f}=\dot{\boldsymbol{r}}+a \boldsymbol{\gamma} \times \boldsymbol{\omega}=0,
$$

а лагранжиан можно представить в форме

$$
\begin{gathered}
L=\frac{1}{2} m \dot{\boldsymbol{r}}^{2}+m(\dot{\boldsymbol{r}}, \boldsymbol{\Omega} \times \boldsymbol{r})+\frac{1}{2} I \boldsymbol{\omega}^{2}+I(\boldsymbol{\omega}, \boldsymbol{\Omega})-V(\boldsymbol{r}), \\
V(\boldsymbol{r})=U(\boldsymbol{r})-\frac{1}{2} m \Omega^{2}\left(r_{1}^{2}+r_{2}^{2}\right),
\end{gathered}
$$

где $V(\boldsymbol{r})$ - обобщенный потенциал, а $U(\boldsymbol{r})$ - потенциальная энергия внешних сил.

Следует иметь в виду, что в системе (3.4) векторы $\boldsymbol{r}$ и $\boldsymbol{\gamma}$ являются зависимыми. Если поверхность, по которой движется центр шара, определяется уравнением

$$
\Phi(\boldsymbol{r})=0,
$$

то вектор нормали к ней оказывается коллинеарен вектору нормали к поверхности, по которой катится шар, и задается гауссовым отображением

$$
\gamma=-\frac{\nabla \Phi(\boldsymbol{r})}{|\nabla \Phi(\boldsymbol{r})|}
$$

В одних случаях для описания движения удобно пользоваться переменными $\boldsymbol{\omega}, \boldsymbol{\gamma}$ (см. раздел 3.2), а в других - переменными $\boldsymbol{\omega}, \boldsymbol{r}$ (см. раздел 3.3). В первом случае уравнения заведомо обладают геометрическим интегралом $\gamma^{2}=1$, а во втором - интегралом $\Phi(\boldsymbol{r})=0$. 
Уравнения движения в подвижной системе координат $O x_{1} x_{2} x_{3}$, можно записать, пользуясь формализмом работы [7]. В явном виде получим

$$
\frac{d}{d t}\left(\frac{\partial L}{\partial \dot{\boldsymbol{r}}}\right)-\frac{\partial L}{\partial \boldsymbol{r}}=\boldsymbol{\lambda}, \quad \frac{d}{d t}\left(\frac{\partial L}{\partial \boldsymbol{\omega}}\right)-\boldsymbol{\omega} \times \frac{\partial L}{\partial \boldsymbol{\omega}}=\boldsymbol{a} \times \boldsymbol{\lambda},
$$

где $\boldsymbol{\lambda}$ - неопределенные множители, представляющие собой реакцию связи (3.2).

Из первого уравнения находим

$$
\boldsymbol{\lambda}=m \ddot{\boldsymbol{r}}+2 m \boldsymbol{\Omega} \times \dot{\boldsymbol{r}}+\frac{\partial V}{\partial \boldsymbol{r}}, \quad \boldsymbol{\Omega}=(0,0, \Omega) .
$$

Подставляя найденное значение $\boldsymbol{\lambda}$ во второе уравнение и воспользовавшись связью (3.2), получим

$$
\begin{gathered}
\widetilde{\mathbf{I}} \dot{\boldsymbol{\omega}}=I \boldsymbol{\omega} \times \boldsymbol{\Omega}+m a^{2}(\boldsymbol{\gamma}, \boldsymbol{\omega}) \dot{\boldsymbol{\gamma}}-2 m a^{2}(\boldsymbol{\gamma}, \boldsymbol{\Omega}) \boldsymbol{\gamma} \times \boldsymbol{\omega}-a \boldsymbol{\gamma} \times \frac{\partial V}{\partial \boldsymbol{r}}, \\
\dot{\boldsymbol{r}}=a \boldsymbol{\omega} \times \boldsymbol{\gamma},
\end{gathered}
$$

где $\widetilde{\mathbf{I}}=\left(I+m a^{2}\right) \mathbf{E}-m a^{2} \boldsymbol{\gamma} \otimes \boldsymbol{\gamma}-$ тензор инерции относительно точки контакта, а $\mathbf{E}-$ единичная матрица.

Вследствие того, что связь (3.2) является однородной по скоростям, рассмотренная система сохраняет энергию. Действительно, выполнив преобразование Лежандра

$$
E=\left(\dot{\boldsymbol{r}}, \frac{\partial L}{\partial \dot{\boldsymbol{r}}}\right)+\left(\boldsymbol{\omega}, \frac{\partial L}{\partial \boldsymbol{\omega}}\right)-L
$$

с учетом связи (3.2), находим интеграл Якоби в форме

$$
E=\frac{1}{2}(\boldsymbol{\omega}, \widetilde{\mathbf{I}} \boldsymbol{\omega})+V(\boldsymbol{r}), \quad V(\boldsymbol{r})=U(\boldsymbol{r})-\frac{1}{2} m \Omega^{2}\left(r_{1}^{2}+r_{2}^{2}\right) .
$$

Кроме того, система (3.4) обладает инвариантной мерой

$$
|\nabla \Phi(\boldsymbol{r})| d \boldsymbol{r} d \boldsymbol{\omega}
$$

и, следовательно, для ее интегрируемости, по теореме Эйлера - Якоби, не хватает двух первых интегралов.

\section{2. Качение шара на поверхности вращения}

Эта задача в наиболее общей форме впервые была рассмотрена в [4] без явного указания интеграла Якоби. То, что он существует при дополнительных ограничениях (типа малости угловой скорости вращения, что, как видно из предыдущих рассуждений, не является существенным), было предсказано в [1]. Явная форма этого интеграла в [1] также не была приведена, однако наличие этого интеграла позволяет более детально исследовать движение.

Представим систему (3.4) в переменных $\boldsymbol{\omega}, \boldsymbol{\gamma}$ в форме

$$
\begin{gathered}
\widetilde{\mathbf{I}} \dot{\boldsymbol{\omega}}=I \boldsymbol{\omega} \times \boldsymbol{\Omega}+m a^{2}(\boldsymbol{\gamma}, \boldsymbol{\omega}) \dot{\boldsymbol{\gamma}}+2 m a^{2}(\boldsymbol{\gamma}, \boldsymbol{\Omega}) \boldsymbol{\omega} \times \boldsymbol{\gamma}+\left(\left(\boldsymbol{\Gamma}^{-1}\right)^{T} \frac{\partial V}{\partial \boldsymbol{\gamma}}\right) \times \gamma, \\
\dot{\boldsymbol{\gamma}}=\boldsymbol{\Gamma}^{-1}(\boldsymbol{\omega} \times \boldsymbol{\gamma}),
\end{gathered}
$$

где $\boldsymbol{\Gamma}$ - матрица с элементами $\Gamma_{i j}=\left\|\frac{1}{a} \frac{\partial r_{i}}{\partial \gamma_{j}}\right\|$. 
Рассмотрим случай, когда поверхность, по которой катится шар, является осесимметричной (ось вращения совпадает с осью симметрии) и ее гауссова кривизна всюду одного знака. Тогда уравнение (3.3) можно однозначно разрешить относительно $\boldsymbol{r}=\boldsymbol{r}(\boldsymbol{\gamma})$ :

$$
\begin{gathered}
r_{1}=f\left(\gamma_{3}\right) \gamma_{1}, \quad r_{2}=f\left(\gamma_{3}\right) \gamma_{2}, \quad r_{3}=\int g\left(\gamma_{3}\right) d \gamma_{3}, \\
g\left(\gamma_{3}\right)=f\left(\gamma_{3}\right)-\frac{1-\gamma_{3}^{2}}{\gamma_{3}} f^{\prime}\left(\gamma_{3}\right),
\end{gathered}
$$

где $f\left(\gamma_{3}\right)$ - функция, параметризующая осесимметричную поверхность (см. подробнее [4]).

Будем полагать, что в (3.5) потенциал внешних сил также инвариантен относительно вращений, то есть $U=U\left(\gamma_{3}\right)$. Тогда система (3.5) обладает полем симметрий

$$
\boldsymbol{u}=\omega_{1} \frac{\partial}{\partial \omega_{2}}-\omega_{2} \frac{\partial}{\partial \omega_{1}}+\gamma_{1} \frac{\partial}{\partial \gamma_{2}}-\gamma_{2} \frac{\partial}{\partial \gamma_{1}} .
$$

Возникновение поля $\boldsymbol{u}$ обусловлено инвариантностью системы относительно вращения вокруг оси симметрии.

В работе [4] выполнена редукция по полю $\boldsymbol{u}$, с помощью которой найдены два линейных по скоростям интеграла вида

$$
F_{i}=f\left(\gamma_{3}\right) v_{1}^{(i)}\left(\gamma_{3}\right)(\boldsymbol{\omega}, \gamma)+v_{2}^{(i)}\left(\gamma_{3}\right) \omega_{3}+v_{3}^{(i)}\left(\gamma_{3}\right), \quad i=1,2,
$$

где функции $v_{1}^{(i)}, v_{2}^{(i)}, v_{3}^{(i)}$, входящие в определение интегралов, представляют собой пару линейно независимых решений системы линейных уравнений:

$$
\begin{gathered}
\frac{d v_{1}}{d \gamma_{3}}=-\frac{v_{2}}{A^{2} f\left(\gamma_{3}\right)}, \quad \frac{d v_{2}}{d \gamma_{3}}=-\frac{f^{\prime}\left(\gamma_{3}\right)}{\gamma_{3}} v_{1}, \\
v_{3}=\frac{\Omega}{a} \int g\left(\gamma_{3}\right)\left(f\left(\gamma_{3}\right) v_{1}-\frac{\gamma_{3}}{A^{2}} v_{2}\right) d \gamma_{3}, \quad A^{2}=1+\frac{I}{m a^{2}} .
\end{gathered}
$$

Таким образом, согласно теореме Эйлера-Якоби система (3.5) является интегрируемой.

Из интеграла Якоби с помощью линейных интегралов (3.6) можно получить явную квадратуру $\gamma_{3}$ (или $\theta$ )

$$
\dot{\gamma}_{3}^{2}=s\left(\gamma_{3}, c_{1}, c_{2}, h\right),
$$

где $c_{1}, c_{2}, h-$ константы первых интегралов (3.6) и интеграла Якоби.

Уравнение (3.8) анализируется стандартными методами [35] и в общем случае, как и при $\Omega=0$, движение носит либо колебательный, либо асимптотический характер, а траектория всегда будет находиться в полосе, ограниченной двумя горизонтальными плоскостями (например, в поле тяжести). Анализ бифуркаций двумерных торов и других качественных свойств движения может быть выполнен аналогично [19]. Топологический анализ трехмерных многообразий (возникающих при исследовании абсолютной динамики) пока не выполнен.

В [4] приведены различные примеры поверхностей, для которых решение (3.7) представляется в элементарных функциях. Простейшим случаем является движение по вращающейся сфере, отмеченное еще Раусом. Далее перейдем к рассмотрению еще одного случая, в котором решение (3.7) при $\Omega=0$ можно также найти в элементарных функциях. 
Качение по поверхности Бельтрами. ${ }^{1}$ Существует несколько необычная механическая демонстрация (которую мы впервые увидели в Математическом институте РАН им. В.А. Стеклова): по внутренней поверхности вертикально расположенной псевдосферы запускают шарик (или монету) с начальной скоростью, направленной под незначительным углом к горизонту (см. рис. 3). Шарик набирает достаточно большую скорость вскоре после начала движения и долгое время катается по некоторой горизонтальной окружности, сохраняя практически одну и ту же минимальную высоту; затем, растеряв энергию (вследствие проскальзывания при больших скоростях), он в конце концов резко падает вниз. Теперь рассмотрим, не ограничиваясь условием $\Omega=0$, какие именно характерные движения шара описывает неголономная модель.

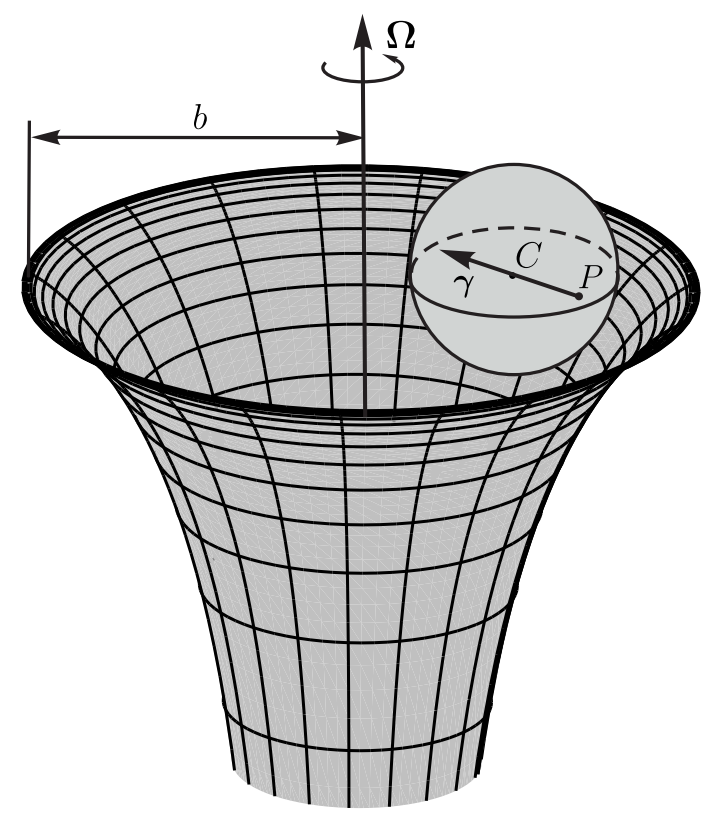

Рис. 3. Шар на поверхности Бельтрами.

Уравнение поверхности Бельтрами (см. рис. 3) в декартовых координатах $(x, y, z)$ можно представить в параметрической форме [8]

$$
x=b \sin \theta \cos \varphi, \quad y=b \sin \theta \sin \varphi, \quad z=b \ln \left(\operatorname{tg} \frac{\theta}{2}\right)+b \cos \theta,
$$

где $\varphi \in[0,2 \pi], \theta \in(0, \pi / 2]$. Ее гауссова кривизна $k$ равна $-b^{-2}$.

Пользуясь (3.9), находим

$$
f\left(\gamma_{3}\right)=-\frac{(a+b) \gamma_{3}}{\sqrt{1-\gamma_{3}^{2}}}, \quad r_{3}=-\frac{a+b}{2} \ln \left(\frac{1+\sqrt{1-\gamma_{3}^{2}}}{1-\sqrt{1-\gamma_{3}^{2}}}\right)+(a+b) \sqrt{1-\gamma_{3}^{2}} .
$$

Потенциал поля тяжести имеет вид

$$
U=m g r_{3},
$$

где g - ускорение свободного падения.

\footnotetext{
${ }^{1}$ Поверхность Бельтрами - это осесимметричная двумерная поверхность постоянной кривизны (псевдосфера), вложенная в трехмерное евклидово пространство.
} 
Характерная траектория точки контакта и зависимости $\omega_{3}$ и $r_{3}$ от времени приведены на рисунках 4 и 5. Как видим из рисунка 4, для приведенной траектории не наблюдается вертикального ухода.

Из рисунка 5 следует, что по мере опускания шара (то есть при уменьшении $r_{3}$ ) возрастает компонента угловой скорости $\omega_{3}$.

Более детальный анализ движения шара можно получить из анализа уравнения (3.8), определяющего гироскопическую функцию, через интегралы (3.6), которые для данного случая можно указать при $\Omega=0$ в элементарных функциях

$$
\begin{aligned}
& F_{1}=\left(\frac{1+\operatorname{tg} \frac{\theta}{2}}{1-\operatorname{tg} \frac{\theta}{2}}\right)^{\frac{1}{A}}\left(\frac{A\left(1-A^{2}\right)+\cos ^{2} \theta \sin \theta}{A+\sin \theta} \omega_{3}+\cos \theta\left(1-\frac{A}{\sin \theta}\right)\left(\gamma_{1} \omega_{1}+\gamma_{2} \omega_{2}\right)\right), \\
& F_{2}=\left(\frac{1-\operatorname{tg} \frac{\theta}{2}}{1+\operatorname{tg} \frac{\theta}{2}}\right)^{\frac{1}{A}}\left(\frac{A\left(1-A^{2}\right)-\cos ^{2} \theta \sin \theta}{A+\sin \theta} \omega_{3}+\cos \theta\left(1+\frac{A}{\sin \theta}\right)\left(\gamma_{1} \omega_{1}+\gamma_{2} \omega_{2}\right)\right), \\
& \gamma_{3}=\cos \theta .
\end{aligned}
$$

Отметим, что аналогичная задача о движении однородной монеты по поверхности Бельтрами является существенно более сложной: в этом случае, видимо, не существует ни дополнительного интеграла движения, ни инвариантной меры.

\section{3. Шар на конусе и плоскости}

Рассмотрим качение шара по поверхности кругового конуса (см. рис. 6). Вследствие нулевой гауссовой кривизны отображение (3.3) вырождается, поэтому для описания динамики воспользуемся переменными $\boldsymbol{\omega}, \boldsymbol{r}$. Координаты центра шара связаны между собой следующим соотношением

$$
r_{3}=\operatorname{tg}(\theta) \sqrt{r_{1}^{2}+r_{2}^{2}}
$$

где $\theta \in[0, \pi / 2)$ - угол раствора конуса. Отсюда нормаль к поверхности находим в форме

$$
\gamma_{1}=-\sin \theta \frac{r_{1}}{\sqrt{r_{1}^{2}+r_{2}^{2}}}, \quad \gamma_{2}=-\sin \theta \frac{r_{2}}{\sqrt{r_{1}^{2}+r_{2}^{2}}}, \quad \gamma_{3}=-\cos \theta
$$

В качестве координат, задающих положение шара на конусе, выберем $\left(r_{1}, r_{2}\right)$; тогда из (3.4) получим замкнутую систему уравнений, описывающих эволюцию $\left(r_{1}, r_{2}, \omega_{1}, \omega_{2}, \omega_{3}\right)$.

Полученная система обладает стандартной инвариантной мерой ( $\rho=$ const) и полем симметрий, которое в этом случае представляется в форме

$$
\boldsymbol{u}=\omega_{1} \frac{\partial}{\partial \omega_{2}}-\omega_{2} \frac{\partial}{\partial \omega_{1}}+r_{1} \frac{\partial}{\partial r_{2}}-r_{2} \frac{\partial}{\partial r_{1}} .
$$

В работе [4] найдены два дополнительных линейных по $\boldsymbol{\omega}$ интеграла

$$
\begin{aligned}
& F_{1}=\omega_{3}+\frac{m R \sin \theta}{I+m R^{2}} \sqrt{r_{1}^{2}+r_{2}^{2}} \Omega, \\
& F_{2}=r_{1} \omega_{1}+r_{2} \omega_{2}+\frac{\sin \theta \sqrt{r_{1}^{2}+r_{2}^{2}}}{\cos \theta} \omega_{3}-\frac{I+2 m R^{2}}{2\left(I+m R^{2}\right)} \frac{\sqrt{r_{1}^{2}+r_{2}^{2}}}{\cos \theta R} \Omega .
\end{aligned}
$$

Таким образом, согласно теореме Эйлера - Якоби система является интегрируемой. 

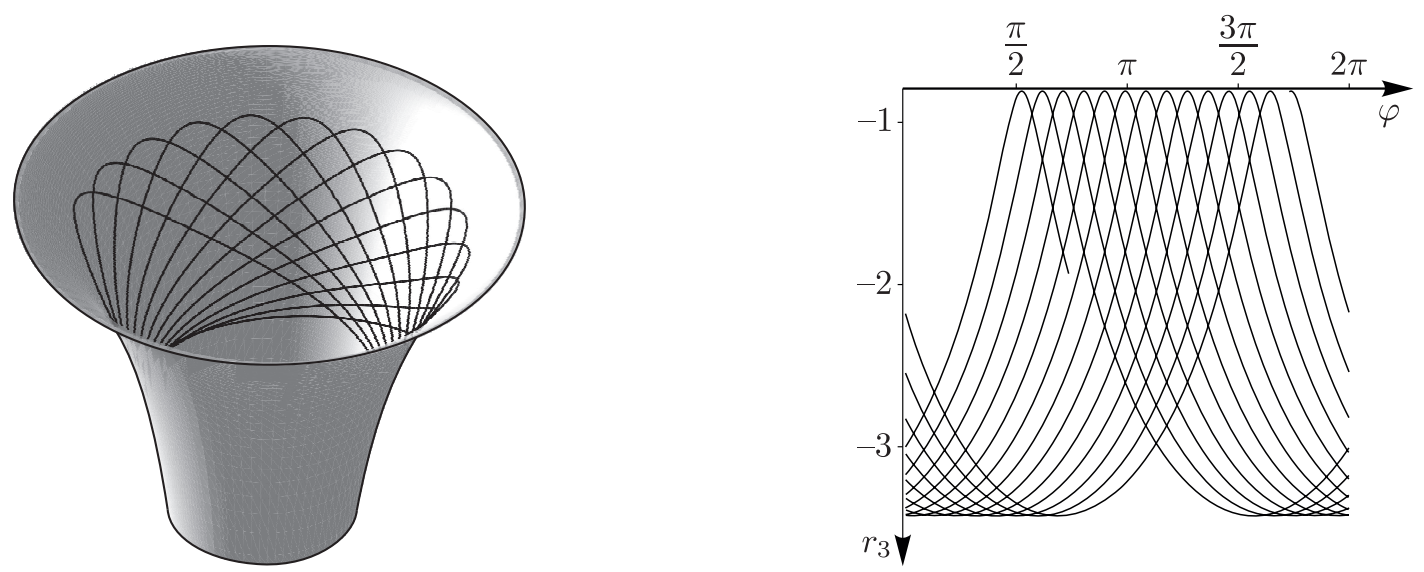

Рис. 4. Траектория движения точки контакта на псевдосфере и ее цилиндрической развертке при параметрах $a=1, b=3, m=1, g=10, I=2 / 5, \Omega_{3}=-1 / 2$ и начальных условиях $\omega_{1}(0)=0.1$, $\omega_{2}(0)=0.5, \omega_{3}(0)=1, \gamma_{1}(0)=-0.52, \gamma_{2}(0)=-0.58, \gamma_{1}(0)=0.62$.
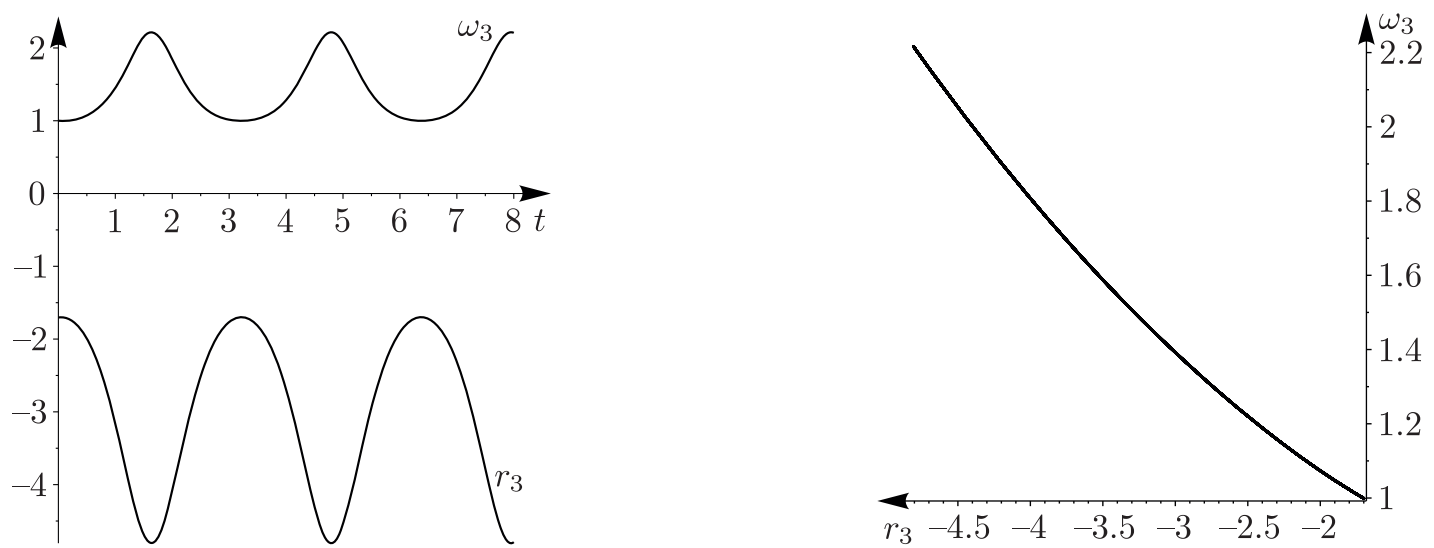

Рис. 5. Графики зависимости $\omega_{3}(t), r_{3}(t)$ и $\omega_{3}\left(r_{3}\right)$ при параметрах $a=1, b=10, m=1, g=10$, $I=2 / 5, \Omega_{3}=-1$ для траектории с начальными условиями $\omega_{1}(0)=0.1, \omega_{2}(0)=0.5, \omega_{3}(0)=1$, $\gamma_{1}(0)=-0.52, \gamma_{2}(0)=-0.58, \gamma_{1}(0)=0.62$.

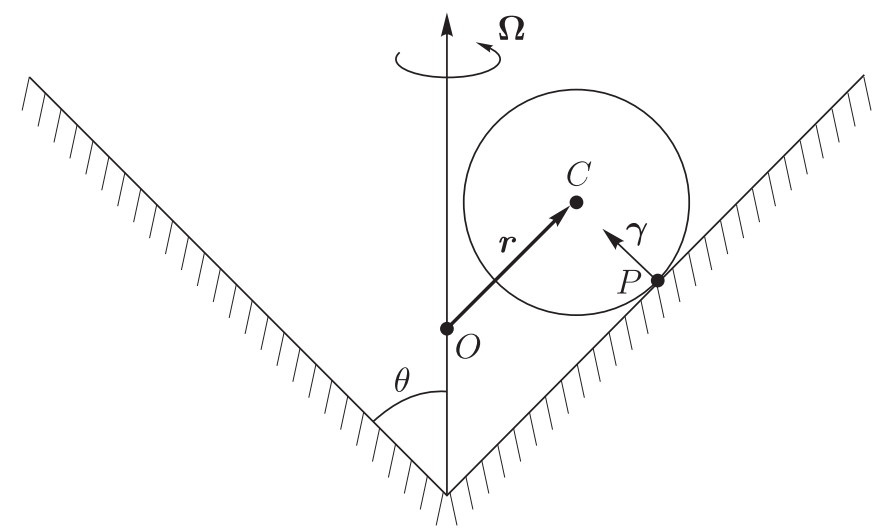

Рис. 6. Шар на конусе. 
В предельном случае $\theta=0$ конус переходит в плоскость, и уравнения движения удобнее рассмотреть в неподвижной системе координат $O x_{o} y_{o} z_{o}$, в которой они представляются в форме

$$
\begin{gathered}
\dot{\omega}_{1}^{(o)}=\operatorname{ma} \Omega\left(a \omega_{2}^{(o)}-y^{(o)} \Omega\right), \quad \dot{\omega}_{2}^{(o)}=-\operatorname{ma} \Omega\left(a \omega_{1}^{(o)}-x^{(o)} \Omega\right), \quad \dot{\omega}_{3}^{(o)}=0, \\
\dot{x}^{(o)}=a \omega_{2}^{(o)}-y^{(o)} \Omega, \quad \dot{y}^{(o)}=-a \omega_{1}^{(o)}+x^{(o)} \Omega,
\end{gathered}
$$

где $\left(x^{(o)}, y^{(o)}\right)$ - координаты точки контакта $P$ шара на плоскости, а $\omega_{\boldsymbol{o}}=\left(\omega_{1}^{(o)}, \omega_{2}^{(o)}, \omega_{3}^{(o)}\right)-$ его угловая скорость.

В этом случае справедливо

Предложение 5. Траектория точки контакта однородного шара на плоскости представляет собой окружность в неподвижной системе координат

$$
\left(x^{(o)}-\frac{a f_{1}}{I \Omega}\right)^{2}+\left(y^{(o)}-\frac{a f_{2}}{I \Omega}\right)^{2}=\mathrm{const},
$$

где $f_{1}$ и $f_{2}$ - биксированные значения первых интегралов (3.10)

$$
F_{1}=\left(I+m a^{2}\right) \omega_{1}^{(o)}-\max ^{(o)} \Omega, \quad F_{2}=\left(I+m a^{2}\right) \omega_{2}^{(o)}-\operatorname{may}^{(o)} \Omega .
$$

Как уже указывалось во введении, результат предложения 5 был известен уже Ирншоу [21], многократно переоткрывался и до сих пор используется для впечатляющих демонстраций из курса теоретической механики.

Отметим работы $[40,41]$, в которых также рассмотрен однородный шар на вращающейся плоскости в связи со сравнением неголономной и вакономной модели качения. Авторы указанных работ приходят к выводу, что неголономная модель лучше согласуется с экспериментальными данными.

В качестве обобщения рассмотренных задач в заключение отметим, что интересно проанализировать динамику, например, однородного шара на вращающейся поверхности (в частности для осесимметричной поверхности, вращающейся вокруг оси симметрий), когда ось вращения наклонена к полю тяжести. Как известно [24, 27, 29], для шара, катящегося по вращающемуся столу, наклон стола приводит к дрейфу шара в перпендикулярном к полю тяжести направлении (аналогично динамике заряженной частицы в электрическом и магнитном полях [23]). Сани Чаплыгина на неподвижной наклонной плоскости изучались в работе [13].

Другой задачей, решенной только в частных случаях, является исследование динамики шара на поверхностях, свободно вращающихся вокруг неподвижной оси. В случае сферы задача восходит к Раусу [9] (в [4] дано явное преобразование, сводящее систему к квадратурам), случай плоскости разбирается в [28]. В общей постановке задача представляет собой частный случай неголономной связки двух твердых тел, рассмотренной в работе [39].

Авторы благодарят В. В. Козлова за плодотворные обсуждения, а также А. В. Цыганова, обратившего внимание авторов на недавнюю работу [1]. 


\section{Список литературы}

[1] Fassò F., Sansonetto N. Conservation of «moving» energy in nonholonomic systems with affine constraints and integrability of spheres on rotating surfaces: Preprint, arXiv:1503.06661 (2015).

[2] Fassò F., Sansonetto N. Conservation of energy and momenta in nonholonomic systems with affine constraints: Preprint, arXiv:1505.01172 (2015).

[3] García-Naranjo L.C., Maciejewski A. J., Marrero J. C., Przybylska M. The inhomogeneous Suslov problem, Phys. Lett. A, 2014, vol. 378, nos. 32-33, pp. 2389-2394.

[4] Borisov A. V., Mamaev I. S., Kilin A. A. The rolling motion of a ball on a surface: New integrals and hierarchy of dynamics, Regul. Chaotic Dyn., 2002, vol. 7, no. 2, pp. 201-219.

[5] Neimark Ju. I., Fufaev N.A. Dynamics of nonholonomic systems, Trans. Math. Monogr., vol. 33, Providence, R.I.: AMS, 1972.

[6] Kozlov V. V. On the existence of an integral invariant of a smooth dynamic system, J. Appl. Math. Mech., 1987, vol.51, no. 4, pp. 420-426; see also: Prikl. Mat. Mekh., 1987, vol.51, no. 4, pp. 538-545.

[7] Borisov A. V., Mamaev I. S. Conservation laws, hierarchy of dynamics and explicit integration of nonholonomic systems, Regul. Chaotic Dyn., 2008, vol. 13, no. 5, pp. 443-490.

[8] Rashevskii P. K. A course in differential geometry, 4th ed., Moscow: Editorial URSS, 2003 (Russian).

[9] Routh E. J. The Advanced part of a treatise on the dynamics of a system of rigid bodies: Being part II of a treatise on the whole subject, 6th ed., New York: Dover, 1955.

[10] Borisov A. V., Kilin A. A., Mamaev I. S. Hamiltonicity and integrability of the Suslov problem, Regul. Chaotic Dyn., 2011, vol. 16, nos. 1-2, pp. 104-116.

[11] Bolsinov A.V., Borisov A. V., Mamaev I.S. Geometrisation of Chaplygin's reducing multiplier theorem, Nonlinearity, 2015 (in press).

[12] Carathéodory C. Der Schlitten, Z. Angew. Math. Mech., 1933, vol. 13, no. 2, pp. 71-76.

[13] Borisov A. V., Mamaev I. S. The dynamics of a Chaplygin sleigh, J. Appl. Math. Mech., 2009, vol. 73, no. 2, pp. 156-161; see also: Prikl. Mat. Mekh., 2009, vol. 73, no. 2, pp. 219-225.

[14] Bolsinov A.V., Borisov A.V., Mamaev I. S. Rolling of a ball without spinning on a plane: The absence of an invariant measure in a system with a complete set of integrals, Regul. Chaotic Dyn., 2012, vol. 17, no. 6, pp. 571-579.

[15] Bizyaev I. A. Nonintegrability and obstructions to the Hamiltonianization of a nonholonomic Chaplygin top, Dokl. Math., 2014, vol.90, no.2, pp.631-634; see also: Dokl. Akad. Nauk, 2014, vol. 458, no. 4, pp. 398-401.

[16] Bolsinov A.V., Borisov A.V., Mamaev I.S. Hamiltonization of nonholonomic systems in the neighborhood of invariant manifolds, Regul. Chaotic Dyn., 2011, vol. 16, no. 5, pp. 443-464.

[17] Bottema O. On the small vibrations of nonholonomic systems, Indag. Math., 1949, vol. 11, pp. $296-298$.

[18] Borisov A. V., Mamaev I. S., Bizyaev I. A. The hierarchy of dynamics of a rigid body rolling without slipping and spinning on a plane and a sphere, Regul. Chaotic Dyn., 2013, vol. 18, no. 3, pp. 277-328.

[19] Borisov A. V., Mamaev I. S., Kilin A. A. Dynamics of rolling disk, Regul. Chaotic Dyn., 2003, vol. 8, no. 2, pp. 201-212.

[20] Borisov A. V., Mamaev I. S. Rolling of a rigid body on plane and sphere: Hierarchy of dynamics, Regul. Chaotic Dyn., 2002, vol. 7, no. 2, pp. 177-200.

[21] Earnshaw S. Dynamics, or An elementary treatise on motion, 3rd ed. Cambridge: Deighton, 1844.

[22] Milne E. A. Vectorial mechanics, New York: Interscience, 1948.

[23] Burns J. A. Ball rolling on a turntable: Analog for charged particle dynamics, Amer. J. Phys., 1981, vol. 48, no. 1, pp. 56-58.

[24] Ehrlich R., Tuszynski J. Ball on a rotating turntable: Comparison of theory and experiment, Amer. J. Phys., 1995, vol. 63, no. 4, pp. 351-359.

[25] Gersten J., Soodak H., Tiersten M. S. Ball moving on stationary or rotating horizontal surface, Amer. J. Phys., 1992, vol. 60, no. 1, pp. 43-47.

[26] Sokirko A. V., Belopolskii A. A., Matytsyn A. V., Kossakowski D. A. Behavior of a ball on the surface of a rotating disk, Amer. J. Phys., 1994, vol. 62, no. 2, pp. 151-156. 
[27] Soodak H., Tiersten M.S. Perturbation analysis of rolling friction on a turntable, Amer. J. Phys., 1996, vol. 64, no. 9, pp. 1130-1139.

[28] Weckesser W. A ball rolling on a freely spinning turntable, Amer. J. Phys., 1997, vol.65, no.8, pp. $736-738$.

[29] Weltner K. Stable circular orbits of freely moving balls on rotating discs, Amer. J. Phys., 1979, vol. 47, no. 11, pp. 984-986.

[30] Fufaev N. A. Rolling of a heavy homogeneous ball over a rough sphere rotating around a vertical axis, Soviet Appl. Mech., 1987, vol. 23, no.1, pp.86-89; see also: Prikl. Mekh., 1987, vol. 23, no. 1, pp. 98-101.

[31] Fufaev N. A. A sphere rolling on a horizontal rotating plane, J. Appl. Math. Mech., 1983, vol.47, no. 1, pp. 27-29; see also: Prikl. Mat. Mekh., 1983, vol. 47, no. 1, pp.43-47.

[32] Tokieda T. Roll models, Amer. Math. Monthly, 2013, vol. 120, no. 3, pp. 265-282.

[33] Kozlov V.V. On the integration theory of equations of nonholonomic mechanics, Regul. Chaotic Dyn., 2002, vol. 7, no. 2, pp. 191-176.

[34] Hermans J. A symmetric sphere rolling on a surface, Nonlinearity, 1995, vol. 8, no. 4, pp. 493-515.

[35] Borisov A. V., Mamaev I.S. Dynamics of a rigid body: Hamiltonian methods, integrability, chaos, 2nd ed., Izhevsk: Institute of Computer Science, 2005 (Russian).

[36] Fedorov Yu. N., Kozlov V. V. Various aspects of $n$-dimensional rigid body dynamics, Amer. Math. Soc. Transl. Ser. 2, 1995, vol.168, pp. 141-171.

[37] Vierkandt A. Über gleitende und rollende Bewegung, Monatsh. Math. Phys., 1892, vol. 3, no. 1, pp. 31-38, 97-116.

[38] Ferrario C., Passerini A. Rolling rigid bodies and forces of constraint: An application to affine nonholonomic systems, Meccanica, 2000, vol. 35, no. 5, pp. 433-442.

[39] Borisov A. V., Kilin A. A., Mamaev I. S. Rolling of a homogeneous ball over a dynamically asymmetric sphere, Regul. Chaotic Dyn., 2011, vol. 16, no. 5, pp. 465-483.

[40] Lewis A. D., Murray R. M. Variational principles for constrained systems: Theory and experiment // Int. J. Non-Linear Mech., 1995, vol. 30, no. 6, pp. 793-815.

[41] Favretti M. Equivalence of dynamics for nonholonomic systems with transverse constraints // J. Dyn. Diff. Equat., 1998, vol. 10, no. 4, pp. 511-536.

\title{
The Jacobi integral in nonholonomic mechanics
}

\author{
Alexey V. Borisov ${ }^{1}$, Ivan S. Mamaev², Ivan A. Bizyaev ${ }^{3}$ \\ ${ }^{1,2}$ Moscow Institute of Physics and Technology (State University) \\ 9 Institutskiy per., Dolgoprudny, Moscow, 141700, Russia \\ ${ }^{3}$ Udmurt State University \\ Universitetskaya 1, Izhevsk, 426034, Russia \\ ${ }^{1}$ borisov@rcd.ru, ${ }^{2}$ mamaev@rcd.ru, ${ }^{3}$ bizaev_90@mail.ru
}

In this paper we discuss conditions for the existence of the Jacobi integral (that generalizes energy) in systems with inhomogeneous and nonholonomic constraints. As an example, we consider in detail the problem of motion of the Chaplygin sleigh on a rotating plane and the motion of a dynamically symmetric ball on a uniformly rotating surface. In addition, we discuss illustrative mechanical models based on the motion of a homogeneous ball on a rotating table and on the Beltrami surface.

MSC 2010: 70F25, 37J60, 70E18

Keywords: nonholonomic constraint, Jacobi integral, Chaplygin sleigh, rotating table, Suslov problem

Received April 28, 2015, accepted May 13, 2015

Citation: Rus. J. Nonlin. Dyn., 2015, vol. 11, no. 2, pp. 377-396 (Russian) 